\title{
Matrix Metalloproteinase-8 Augments Bacterial Clearance in a Juvenile Sepsis Model
}

\author{
Sarah J Atkinson, ${ }^{1,2}$ Brian M Varisco, ${ }^{1,3}$ Mary Sandquist, ${ }^{1,3}$ Meghan N Daly, ${ }^{1,2}$ Lindsey Klingbeil, ${ }^{1,2}$ \\ Joshua W Kuethe, ${ }^{2,4}$ Emily F Midura, ${ }^{2,4}$ Kelli Harmon, ${ }^{1}$ Amy Opoka, ${ }^{1}$ Patrick Lahni, ${ }^{1}$ Giovanna Piraino, ${ }^{1}$ \\ Paul Hake, ${ }^{1}$ Basilia Zingarelli, ${ }^{1,3}$ Joel E Mortensen, ${ }^{5}$ James L Wynn, ${ }^{6}$ and Hector $R$ Wong ${ }^{1,3}$
}

${ }^{1}$ Division of Critical Care Medicine, Cincinnati Children's Hospital Medical Center and Cincinnati Children's Research Foundation, Cincinnati, Ohio, United States of America; ${ }^{2}$ Department of Surgery, University of Cincinnati College of Medicine; ${ }^{3}$ Department of Pediatrics, University of Cincinnati College of Medicine; ${ }^{4}$ Division of Research, Department of Surgery, University of Cincinnati College of Medicine; ${ }^{5}$ Department of Pathology and Laboratory Medicine, Cincinnati Children's Hospital Medical Center; and ${ }^{6}$ Department of Pediatrics, University of Florida, Gainesville, Florida, United States of America

\begin{abstract}
Genetic ablation or pharmacologic inhibition of matrix metalloproteinase-8 (MMP8) improves survival in an adult murine sepsis model. Because developmental age influences the host inflammatory response, we hypothesized that developmental age influences the role of MMP8 in sepsis. First, we compared sepsis survival between wild-type (WT, C57BL/6) and MMP8 null juvenile-aged mice (12-14 d) after intraperitoneal injection of a standardized cecal slurry. Second, peritoneal lavages collected $6 \mathrm{~h}$ and $18 \mathrm{~h}$ after cecal slurry injection were analyzed for bacterial burden, leukocyte subsets and inflammatory cytokines. Third, juvenile WT mice were pretreated with an MMP8 inhibitor prior to cecal slurry injection; analysis of their bacterial burden was compared with vehicle-injected animals. Fourth, the phagocytic capacity of WT and MMP8 null peritoneal macrophages was compared. Finally, peritoneal neutrophil extracellular traps (NETs) were compared using immunofluorescent imaging and quantitative image analysis. We found that juvenile MMP8 null mice had greater mortality and higher bacterial burden than WT mice. Leukocyte counts and cytokine concentrations in the peritoneal fluid were increased in the MMP8 null mice relative to the wild-type mice. Peritoneal macrophages from MMP8 null mice had reduced phagocytic capacity compared to WT macrophages. There was no quantitative difference in NET formation, but fewer bacteria were adherent to NETs from MMP8 null animals. In conclusion, in contrast to septic adult mice, genetic ablation of MMP8 increased mortality following bacterial peritonitis in juvenile mice. This increase in mortality was associated with reduced bacterial clearance and reduced NET efficiency. We conclude that developmental age influences the role of MMP8 in sepsis. Online address: http://www.molmed.org
\end{abstract}

doi: $10.2119 /$ molmed.2016.00058

\section{INTRODUCTION}

The incidence of sepsis in the United States continues to rise despite antibiotics and vaccines (1). Sepsis is associated with high morbidity and mortality among adult and pediatric populations, and among the latter, the highest burden is seen in younger children and infants $(2,3)$.
Therefore, there is a need to better define the critical signaling pathways in sepsis and identify novel therapeutic targets that are age- and context-appropriate.

MMP8 is one of the highest expressed genes in children with sepsis and contributes to multiple gene networks involved in innate immunity and

Address correspondence to Hector R Wong, Division of Critical Care Medicine-MLC 2005, Cincinnati Children's Hospital Medical Center, 3333 Burnet Avenue, Cincinnati, OH 45229, USA. Phone: 513-636-4359; Fax: 513-636-4267; E-mail: hector.wong@cchmc.org. Submitted March 7, 2016; Accepted for Publication July 22, 2016; Published Online (www.molmed.org) August 8, 2016.

inflammation (4-8). Increased MMP8 mRNA and protein expression is associated with increased risk of poor outcome among children with septic shock, but cause and effect are not established $(8,9)$. In addition, MMP8 gene variants are associated with differential cytokine responses among normal volunteers challenged with endotoxin (10) and with preterm premature rupture of membranes (11), which is often the result of intrauterine infection.

Based on these data, we have been interested in exploring MMP8 as a candidate novel therapeutic target in sepsis. MMP8 was originally identified as a neutrophil product (neutrophil collagenase) but has since been found in numerous cell types, including activated macrophages, smooth muscle cells 
and endothelial cells (12). Its role in chemotaxis, neutrophil cell migration, chemokine modulation and cleavage of nonmatrix proteins added to our initial interest in MMP8 as a therapeutic target for sepsis (12-14).

Our initial investigations of the mechanistic role of MMP8 in sepsis utilized cecal ligation and puncture (CLP) in an adult murine model and showed that genetic ablation or pharmacologic inhibition of MMP8 improves survival following CLP (8). To further delineate the role of MMP8 in sepsis, we utilized alternative sepsis models in adult mice and found that intestine-derived MMP8 is a critical component of septic peritonitis secondary to intestinal compromise (15).

While these data support further exploration of MMP8 as a therapeutic target, we cannot assume that the data are germane to children, because developmental age strongly influences the host response to sepsis $(5,8)$. In contrast to adult mice, adaptive immunity contributes minimally to murine neonatal sepsis survival following cecal slurry injection (16). Recently, genome-wide expression patterns were compared among neonates, infants, toddlers and schoolage children with septic shock and found to differ significantly by age group, with the greatest differences seen among neonates (5). Therefore, the role of MMP8 in sepsis may vary depending on the developmental age of the host, as well as the sepsis model used.

Here we seek to further define the mechanism by which MMP8 influences outcomes in sepsis by employing a juvenile sepsis model that uses intraperitoneal injections of a standardized cecal slurry in 2-wk-old mice. We test the hypothesis that the role of MMP8 in sepsis is dependent on developmental age.

\section{MATERIALS AND METHODS}

\section{Murine Models}

All aspects of this study complied with the Guide for the Care and Use of Laboratory Animals by the National Academies of Sciences, Engineering, and Medicine Institute for Laboratory Animal Research and Division on Earth and Life Studies (17) and met the approval of our Institutional Animal Care and Use Committee. MMP8 null mice on a C57BL/ 6 background were provided by Dr. Steven Shapiro, University of Pittsburgh. Wild-type C57BL/6 mice were obtained from Charles Rivers Laboratories. All mice were fed standard rodent chow and maintained on 12-h light-dark cycles.

\section{Cecal Slurry}

We adapted the cecal slurry method from Wynn et al. and prepared a fresh slurry for each experiment using three wild-type C57BL/ 6 female donors ages 6-9 wks (18). Following euthanasia with $\mathrm{CO}_{2}$, a midline laparotomy was made and the cecum excised from each donor. The distal portion of the appendix was removed and cecal contents were collected in a $50 \mathrm{~mL}$ sterile conical tube. Five percent dextrose in water (D5W) was added to bring the stool concentration to $80 \mathrm{mg} / \mathrm{mL}$. The solution was then vortexed and sonicated to create a homogeneous solution. The solution was then passed through a sterile $100-\mu \mathrm{m}$ filter to capture any remaining large particles and ensure that it would pass through a 27 -gauge needle.

Sepsis was induced in mixed-sex juvenile mice (12-14 d) via intraperitoneal injection of the cecal slurry at a dose of $0.5 \mathrm{mg} / \mathrm{g}$ via a 27 -gauge needle. Control mice were injected with an equivalent volume of D5W. Following the cecal slurry injection, animals were monitored for survival (up to $7 \mathrm{~d}$ ) or were euthanized at $6 \mathrm{~h}$ and $18 \mathrm{~h}$ for procurement of biological specimens.

All experimental protocols, except one, were conducted in the absence of antibiotics. The one exception was an experimental protocol in which MMP8 null mice were treated with intraperitoneal metronidazole $(12.5 \mathrm{mg} / \mathrm{kg})$ and ceftriaxone $(25 \mathrm{mg} / \mathrm{kg})$, beginning $6 \mathrm{~h}$ after cecal slurry injection, and then every $12 \mathrm{~h}$, for a total of 6 doses.

\section{Flow Cytometry}

Single-cell suspensions were prepared from peritoneal lavage samples collected $6 \mathrm{~h}$ or $18 \mathrm{~h}$ after intraperitoneal slurry injections. Cell counts were determined using a Coulter AcT 10 cell counter (Beckman Coulter). Cells were suspended in fluorescence-activated cell sorting (FACS) buffer (phosphate buffered saline [PBS] with $1 \%$ bovine albumin and $0.1 \%$ azide) and nonspecific binding to cells was prevented by adding 5\% rat serum (Invitrogen) and $1 \mu \mathrm{L} /$ sample of Fc Block (BD Pharmingen). Cells were then stained with $1 \mu \mathrm{L}$ of stain per sample of rat anti-mouse PerCP Cy5.5 Ly6G (BD Bioscience, clone 1A8), FITC Ly6C (BD Bioscience, clone AL-21), PE F4/80 (Biolegend, clone T45-2342), and APC CD11b (BD Bioscience, clone M1/70). Nonviable cells and lymphocytes were excluded. Myeloid cell populations were identified using standard forward and side scatter parameters. All analyses were performed using Attune software (Life Technologies).

\section{Cytokines}

Peritoneal lavage samples were collected at $6 \mathrm{~h}$ and $18 \mathrm{~h}$ after cecal slurry injections, in an unblinded manner. Samples were collected by removing the skin overlying the peritoneal cavity while keeping the peritoneum intact. With a 27-gauge needle, $500 \mu \mathrm{L}$ sterile PBS was injected into the cavity in the left upper quadrant. Animals were manipulated to distribute the PBS throughout the entire peritoneum. Then $150 \mu \mathrm{L}$ peritoneal fluid was aspirated from the left lower quadrant of the peritoneum and stored at $-80^{\circ} \mathrm{C}$ until the time of analysis. Lavage samples were analyzed for interleukin (IL)-1ß, IL-6, IL-10, macrophage inflammatory protein- $1 \alpha$ (MIP- $1 \alpha$, CCL3), lipopolysaccharide-induced CXC chemokine (LIX), tumor necrosis factor $\alpha$ $(\mathrm{TNF} \alpha)$ and keratinocyte-derived chemokine (CXCL1) using a Luminex multiplex system (Millipore). Results were standardized to total protein content (calculated via Pierce BCA Protein Assay Kit, Thermo Scientific) within each sample. 


\section{Bacterial Clearance}

Animals were euthanized at $18 \mathrm{~h}$ and peritoneal lavage samples were collected as detailed above. Serial dilutions of each lavage were prepared using sterile PBS. Diluted specimens were plated onto 5\% sheep blood agar plates (Becton Dickinson) for the growth and enumeration of aerobic colonies, and Brucella blood agar plates (Becton Dickinson) for the growth and enumeration of anaerobic bacterial colonies. Plates were incubated at $37^{\circ} \mathrm{C}$ in aerobic or anaerobic conditions. Plated dilutions with 30-300 colonies were used for counts.

\section{Pharmacologic MMP8 Inhibitor and Bacterial Clearance}

The MMP8 inhibitor ((3R)-N-hydroxy2-(4-methoxyphenyl)sulfonyl-3,4-dihydro-1H-isoquinoline-3-carboxamide; Santa Cruz Biotech) was dissolved in $1 \%$ DMSO in PBS to a final concentration of $0.03 \mathrm{mg} / \mathrm{mL}$. Animals received a $0.3 \mathrm{mg} / \mathrm{kg}$ dose of inhibitor via intraperitoneal injection with a 27-gauge needle every $12 \mathrm{~h}$ beginning $24 \mathrm{~h}$ prior to slurry injection. Control animals were dosed with an equivalent volume of $1 \%$ dimethyl sulfoxide (DMSO) in PBS. Peritoneal lavage and bacterial clearance were done in an identical fashion to bacterial clearance, as detailed above.

\section{Phagocytosis Activity of Murine Peritoneal Macrophages}

Healthy 12-to-14-d-old MMP8 null and wild-type mice were euthanized with carbon dioxide and exsanguination via cardiac puncture. The peritoneum was exposed and $500 \mu \mathrm{L}$ sterile PBS was injected into the peritoneal space and distributed throughout the cavity. Then $150 \mu \mathrm{L}$ was withdrawn and cells were centrifuged for $10 \mathrm{~min}$ at $153 \mathrm{~g}$. The cell pellet was resuspended in Dulbecco's modified Eagle medium (DMEM; Gibco) to a final concentration of $10^{6}$ cells $/ \mathrm{mL}$. For positive control wells, RAW 264.7 macrophages (ATCC) were cultured in media (RPMI supplemented with $10 \%$ fetal bovine serum, $100 \mathrm{U} / \mathrm{mL}$ penicillin and $0.1 \mathrm{mg} / \mathrm{mL}$ of streptomycin; Invitrogen) to a final concentration of $10^{6}$ cells $/ \mathrm{mL}$.
Negative-control wells were prepared by adding $150 \mu \mathrm{L}$ DMEM to 8 wells on the microplate. For positive-control wells, $10^{5}$ RAW macrophages were added to 16 wells on the microplate. The remaining wells were completed with $10^{5} \mathrm{MMP8}$ null or wild-type primary murine peritoneal macrophages. DMEM media was used to bring the final volume of the positive-control and experimental wells to $150 \mu \mathrm{L}$. The microplate was covered and incubated for $2 \mathrm{~h}$ at $37^{\circ} \mathrm{C}$. DMEM solution was removed with vacuum aspiration and $100 \mu \mathrm{L}$ of $1 \mathrm{mg} / \mathrm{mL}$ Texas Red-tagged E. coli (Life Technologies) suspension was added to all wells. After $2 \mathrm{~h}$, the suspension was aspirated. Using $480 \mathrm{~nm}$ excitation and $520 \mathrm{~nm}$ emission with a Synergy HT plate reader, a net reading was calculated for each individual experiment by subtracting the negative-control values from positive-control and experimental values. Since the experiment was performed multiple times, a correction factor was calculated based on the ratio between average positive-control values from each experiment.

In a variation of these experiments, peritoneal macrophages obtained from wild-type mice were pretreated ex vivo with the MMP8 inhibitor $(0.1 \mathrm{mg} / \mathrm{mL})$ or vehicle ( $1 \%$ DMSO in cell culture media), for $1 \mathrm{~h}$ before exposure to tagged E. coli.

\section{Quantification of NET Formation and Bacterial Entrapment}

Animals (9 per group) were euthanized at $6 \mathrm{~h}$ for peritoneal lavage. Peritoneal lavage samples were immediately pipetted onto glass slides and allowed to dry overnight. Slides were blocked for $2 \mathrm{~h}$ at room temperature in PBS containing 2\% BSA and stained for $1 \mathrm{~h}$ with Sytox Green (Life Biosciences) at 1:10,000 dilution. Slides were washed 3 times with PBS and mounted with ProLong Gold anti-fade reagent without DAPI (Life Technologies). For co-staining studies, anti-neutrophil elastase antibody (1:200 dilution, Santa Cruz) and anti-histone H4 antibody (1:1000 dilution, AbCam) were used with appropriate fluorophore-conjugated secondary antibodies (1:10,000 dilution; Jackson ImmunoResearch). Slides were imaged using a Nikon 90i upright wide-field microscope (3 images per animal).

Net formation was quantified using the Imaris software package (v7.7.1, Bitplane AG, Mathworks). To do so, the total DNA of a field at $10 \times$ magnification was defined as signal above negative control in the green channel. Nuclear DNA was defined as a green signal in the nucleus and was manually masked. Fraction of DNA outside of the nucleus was used as a measure of NET formation.

To quantify bacterial entrapment by NETs, Texas Red-tagged E. coli (Life Technologies) were suspended in the cecal slurry at a concentration of $1 \mu \mathrm{g} / \mathrm{mL}$. The slurry was briefly vortexed and injected intraperitoneally through a 27-gauge needle at a $0.5 \mathrm{mg} / \mathrm{kg}$ dose. Samples were collected $6 \mathrm{~h}$ post-injection. Peritoneal fluid slides were stained with Sytox Green and imaged. For analysis, the bacteria were defined as red signal over negative control and DNA was defined as green signal over negative control. A distance transformation was applied, with bacteria within $1 \mu \mathrm{m}$ of the DNA signal being defined as associated with the NET and greater than $1 \mu \mathrm{m}$ as not associated. To normalize for differences in sample dilution, the fractional coverage of DNA (total DNA area/total image area) was used to create a "predicted" bacteria count that estimated the number of bacteria that would be expected to be associated with the NET by chance. An observed-to-predicted ratio was then calculated for each image for comparison of NET efficiency.

\section{Statistical Analysis}

Statistical analysis was performed using SigmaStat for Windows version 3.10 (SysStat Software). Data are represented as mean \pm SEM or as median with interquartile range of $\mathrm{n}$ observations, where $n$ represents the number of subjects in each group. When comparing 2 groups at the same time point, a Student $t$ test was performed. For multiple group 
comparisons at a single time point, oneway analysis of variance (ANOVA) with Student-Newman-Keuls correction was used. For multiple group analysis at different time points, a 2-way ANOVA with Student-Newman-Keuls correction was performed. If data did not follow a normal distribution, a Mann-Whitney rank sum test or ANOVA on ranks test was performed. For survival analysis, a log rank survival analysis was used. $P$ values less than 0.05 were considered significant.

\section{RESULTS}

\section{Genetic Ablation of MMP8 in Juvenile Mice Increases Mortality Following Sepsis from Polymicrobial Peritonitis}

Figure 1 shows that juvenile MMP8 null mice had greater sepsis mortality compared with the wild-type mice after intraperitoneal cecal slurry injection. This phenotype is in direct contrast to that of adult MMP8 null and wild-type mice (8 to 10 wks of age), which have similar mortality after intraperitoneal cecal slurry injection (15). The increased susceptibility of MMP8 null juvenile mice to peritoneal sepsis suggests that MMP8 plays an important role in the juvenile innate immune response.

\section{Local Cytokine Response in Juvenile Wild-Type and MMP8 Null Mice with Peritoneal Sepsis}

Peritoneal lavage samples at $6 \mathrm{~h}$ and $18 \mathrm{~h}$ post-slurry were analyzed for the cytokines IL-1ß, IL-6, IL-10, MIP-1 $\alpha$ (CCL3), LIX), TNF $\alpha$ ) and CXCL1. At $6 \mathrm{~h}$ after cecal slurry injection, the median concentrations of all cytokines except LIX were significantly higher in the peritoneal lavage samples from MMP8 null juvenile mice, compared with those of WT juvenile mice (Table 1). These differences continued at $18 \mathrm{~h}$ after cecal slurry injection (Table 1). When the cytokine concentrations were normalized to the respective peritoneal fluid protein concentrations, no differences were observed between cytokine concentrations from MMP8 null and wild-type

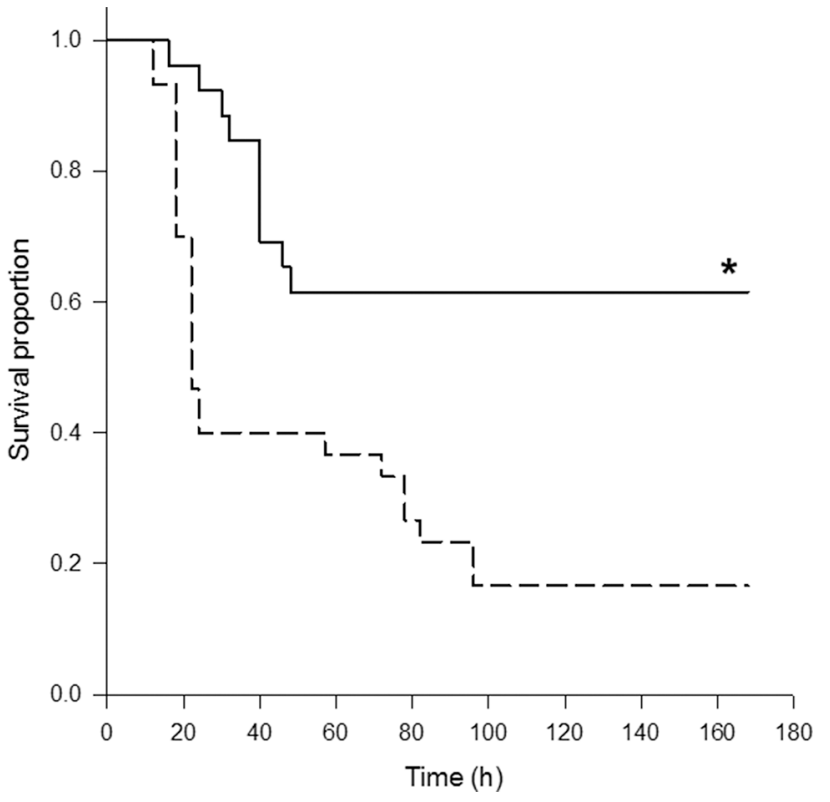

Figure 1. Survival of juvenile MMP8 null and wild-type mice following intraperitoneal cecal slurry injections. Wild-type (solid line, $n=26$ ) and MMP8 null (dashed line, $n=30$ ) juvenile mice (12-14 d) received intraperitoneal injections of cecal slurry at $0.5 \mathrm{mg} / \mathrm{g}$ and were monitored for survival. MMP8 null mice demonstrated decreased survival after cecal slurry injection (experiment performed 4 times). ${ }^{*} p<0.001$ by log rank survival analysis.

Table 1. Cytokine concentrations in peritoneal fluid $6 \mathrm{~h}$ and $18 \mathrm{~h}$ after cecal slurry injection, without correction for total intraperitoneal fluid protein concentration.

\begin{tabular}{|c|c|c|c|}
\hline \multicolumn{4}{|c|}{$6 \mathrm{~h}$} \\
\hline Cytokine & Wild-Type pg/mL (IQR), $\mathrm{n}=18$ & MMP8 null pg/mL (IQR), $\mathrm{n}=21$ & $P$ value \\
\hline ILI $\beta$ & $148(91-186)$ & $242(210-302)$ & $<0.001$ \\
\hline IL6 & $14,874(7273-16,782)$ & $16,6692(15,939-17,834)$ & 0.038 \\
\hline IL 10 & 955 (340-2030) & $2,218(1,417-3,188)$ & 0.006 \\
\hline CCL3 & $572(300-1,461)$ & $1,441(802-2,402)$ & 0.038 \\
\hline LIX & $911(659-1,187)$ & $1,001(667-1,681)$ & 0.291 \\
\hline TNF $\alpha$ & $45(26-70)$ & $76(62-92)$ & 0.014 \\
\hline CXCL1 & $4,872(1,321-15,078)$ & $14,715(6,206-16,474)$ & 0.015 \\
\hline \multicolumn{4}{|c|}{$18 \mathrm{~h}$} \\
\hline & $n=20$ & $n=20$ & \\
\hline $\mathrm{IL} 1 \beta$ & $137(74-249)$ & $288(227-410)$ & 0.020 \\
\hline IL6 & $2,256(429-7,948)$ & $20,285(6,256-20,300)$ & 0.032 \\
\hline IL 10 & $1,031(333-2,718)$ & $1,967(1,145-3,208)$ & 0.306 \\
\hline CCL3 & $541(324-1,230)$ & $1,763(1,102-3,203)$ & 0.028 \\
\hline LIX & $364(114-517)$ & $470(396-687)$ & 0.251 \\
\hline $\mathrm{TNF} \alpha$ & $73(24-113)$ & $112(89-193)$ & 0.067 \\
\hline CXCL1 1 & $1,064(425-2,934)$ & $8,910(3,706-21,701)$ & 0.012 \\
\hline
\end{tabular}

juvenile mice at any time point (data not shown), except for CXCL1 measured at 18 h: 5,049 pg/mL (IQR: 1,706 to 14,092 ) versus 1,283 ng/mL (IQR: 704 to 1,936), respectively $(p=0.019)$. These data suggest that the local cytokine response of juvenile MMP8 null mice is more robust than that of juvenile WT mice after 
intraperitoneal cecal slurry injection. However, the results obtained after correction for total protein indicate that the cytokine data might be confounded by a general increase in total protein concentration within the peritoneal cavity.

\section{Bacterial Clearance Is Impaired in Juvenile MMP8 Null Mice}

At $18 \mathrm{~h}$ after cecal slurry injection, both aerobic (Figure 2A) and anaerobic (Figure 2B) bacterial colony counts were greater in MMP8 null juvenile mice compared with wild-type mice. This suggests that increased mortality in the juvenile MMP8 null mice after cecal slurry injection is due to a reduced ability to clear bacteria. To further explore this possibility, we treated juvenile wild-type and MMP8 null mice with
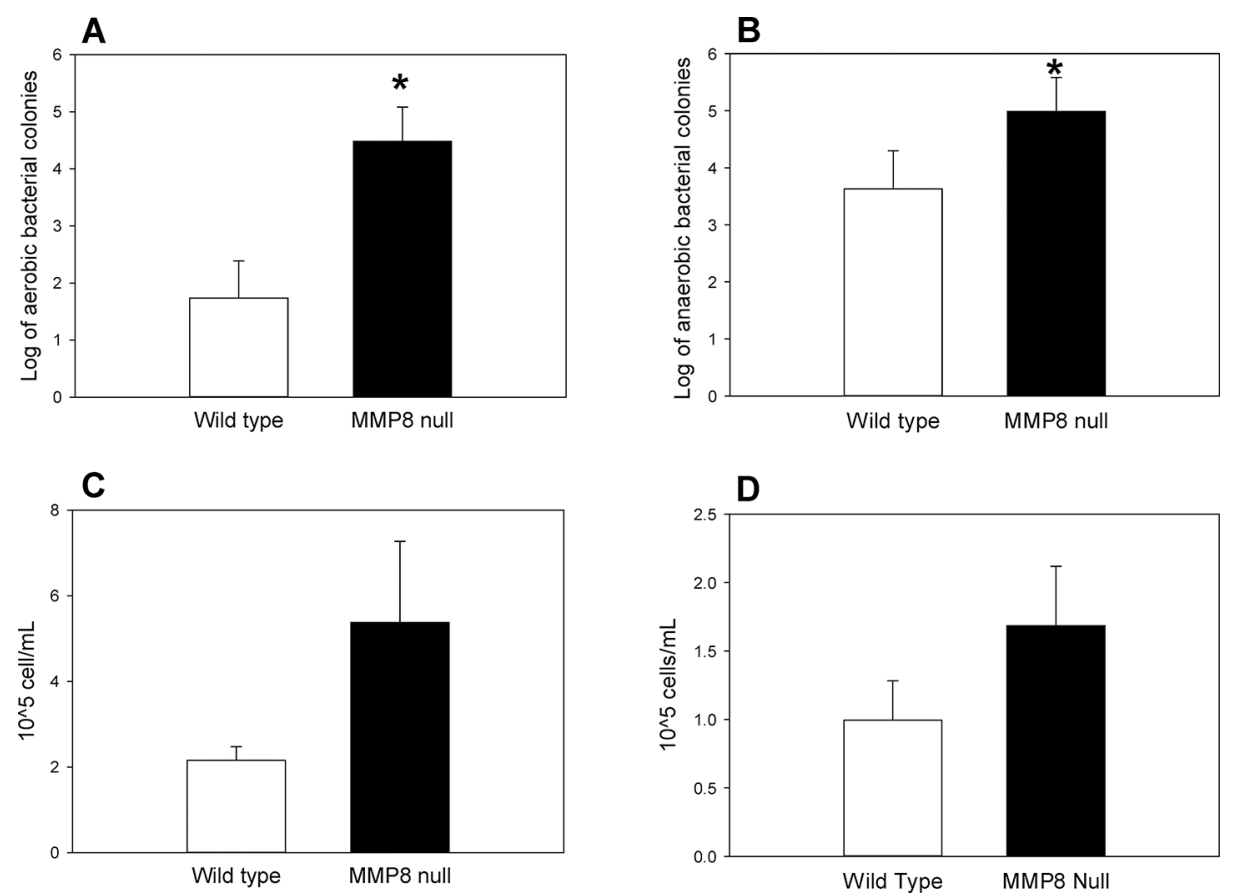

antibiotics beginning at $6 \mathrm{~h}$ after cecal slurry injection, and then every $12 \mathrm{~h}$ for a total of $3 \mathrm{~d}$. We observed no deaths in juvenile wild-type or MMP8 null mice challenged with cecal slurry and subsequently treated with antibiotics. Thus, the increased mortality seen in juvenile MMP8 null mice is eliminated with antibiotic treatment, further supporting the concept that increased mortality is due to a reduced ability to clear bacteria.

To determine if the reduced ability of MMP8 null mice to clear bacteria is due to a quantitative difference in phagocytic cells, we measured the number of neutrophils and macrophages in the peritoneal fluid. At baseline, there were no differences in the number of neutrophils and macrophages between MMP8 null and wild-type mice (data not shown).

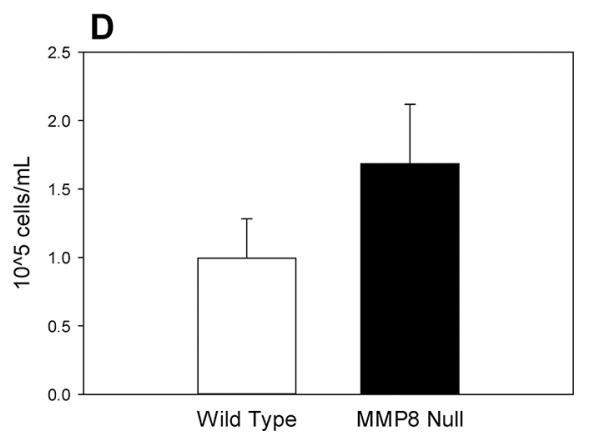

Figure 2. Aerobic and anaerobic bacterial colony counts and neutrophil counts from peritoneal lavage samples after cecal slurry injection. (A) MMP8 null animals $(n=13)$ had a higher aerobic bacterial burden $18 \mathrm{~h}$ after cecal slurry injection, compared with that found in the peritoneal lavage of wild-type animals $(n=10$, experiment performed 2 times). ${ }^{*} p<0.05$ by 2 -tailed $t$ test. (B) Similarly, anaerobic bacterial burden was greater in peritoneal lavage of MMP8 null animals $(n=19) 18 \mathrm{~h}$ after cecal slurry injection compared with that of wild-type animals ( $n=20$, experiment performed 3 times). ${ }^{*} p<0.05$ by 2-tailed $t$ test. (C, D) Neutrophil counts in the corresponding peritoneal lavage samples tended to be higher in MMP8 null mice at $6 \mathrm{~h} \mathrm{(C)}$ and $18 \mathrm{~h}(\mathrm{D})$, but were not statistically different. All data reported as mean \pm SEM.
At $6 \mathrm{~h}$ (Figure 2C) and $18 \mathrm{~h}$ (Figure 2D) after cecal slurry injection, there was a trend toward a greater number of neutrophils in the peritoneal fluid from MMP8 null mice, but this did not reach statistical significance. Similar observations were made for peritoneal macrophages and total white blood cell counts at both the $6 \mathrm{~h}$ and $18 \mathrm{~h}$ time points (data not shown). These data suggest that the increased bacterial counts seen in the peritoneal fluid of $M M P$ null mice are not a reflection of decreased recruitment of phagocytic cells to the peritoneal cavity.

\section{Pharmacologic Inhibition of MMP8 Activity Reduces Bacterial Clearance in Wild-Type Juvenile Mice with Peritoneal Sepsis}

Chronic genetic ablation of MMP8 may cause unidentified developmental impairments or genetic compensation, rendering juvenile mice less capable of clearing bacteria from the peritoneal space, unrelated to MMP8 per se. To test this possibility, we treated wild-type juvenile mice with a pharmacologic inhibitor of MMP8 and challenged them with cecal slurry injection. Figure 3 shows that pharmacologic inhibition of MMP8 in wild-type

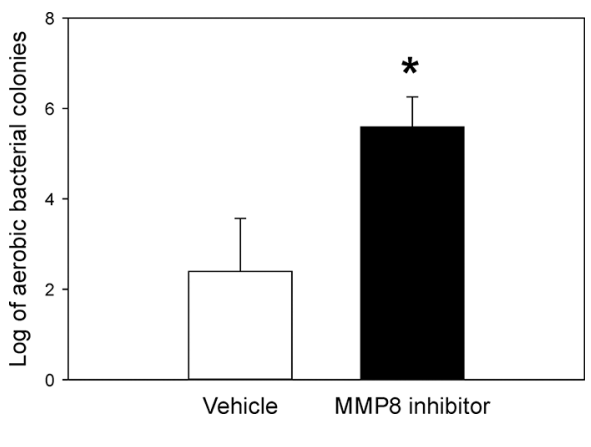

Figure 3. Aerobic bacterial colony counts from juvenile wild-type mice pretreated with a pharmacologic MMP8 inhibitor versus vehicle. Juvenile wild-type mice treated with MMP8 inhibitor $(n=10)$ prior to cecal slurry injection had a higher intraperitoneal bacterial burden compared with mice treated with vehicle only and subjected to cecal slurry injection ( $n=8$, experiment performed twice). ${ }^{*} p<0.05$ by 2 -tailed $t$ test. All data reported as mean \pm SEM. 
juvenile mice subjected to cecal slurry replicated the increased bacterial burden phenotype of MMP8 null mice at $18 \mathrm{~h}$. This further supports a role for MMP8 in juvenile mouse innate immunity.

\section{MMP8 Null Primary Peritoneal Macrophages Have Impaired Phagocytic Activity}

To explore the mechanism of impaired bacterial clearance in MMP8 null juvenile mice, we compared the ex vivo phagocytic function of MMP8 null and wild-type peritoneal macrophages using fluorescently labeled E. coli. These experiments involved peritoneal macrophages from mice not previously challenged with intraperitoneal cecal slurry injections. Figure 4A shows greater intracellular fluorescence in the wild-type peritoneal macrophages compared with the MMP8 null peritoneal macrophages. This indicates that MMP8 null peritoneal macrophages have reduced phagocytic ability relative to their wild-type counterparts.

To corroborate these findings, we treated wild-type peritoneal macrophages with either an MMP8 inhibitor $(0.1 \mathrm{mg} / \mathrm{mL})$ or the DMSO vehicle $(1 \%)$, and exposed them to fluorescently labeled E. coli. Figure 4B shows decreased intracellular fluorescence in the wild-type macrophages treated with the

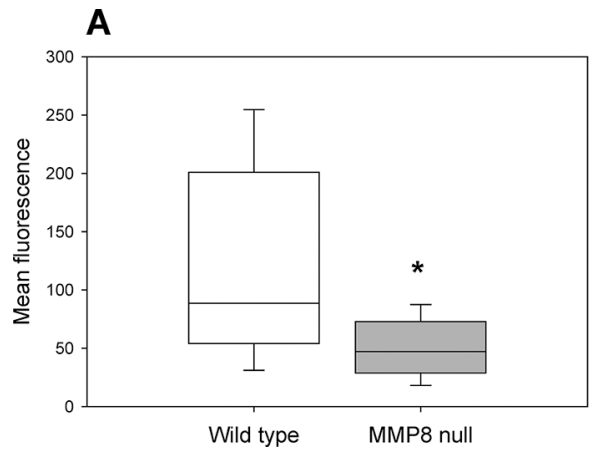

MMP8 inhibitor compared with those treated with the DMSO vehicle. Thus, the reduced bacterial clearance in $M M P 8$ null juvenile mice is due, in part, to impaired phagocytosis.

\section{MMP8 Enhances Bacterial Adherence to NETs but Not NET Formation}

To further define the mechanisms of impaired bacterial clearance seen in the juvenile MMP8 null mice, we harvested peritoneal fluid and quantified NET formation by image analysis. To ensure that our assay was measuring DNA from neutrophils, we tested for colocalization of DNA signal with histone and neutrophil elastase (Figure 5A). While NET formation was increased by cecal slurry relative to baseline, there were no differences in the degree of NET formation between wild-type and MMP8 null animals challenged with cecal slurry (Figure 5B).

To test for differences in NET function, we quantified bacterial adherence to NETs using fluorophore-labeled E. coli suspended in the cecal slurry. Figures 6A and $6 \mathrm{C}$ show representative images of fluorophore-labeled bacteria adhering to NETs of wild-type and MMP8 null mice, respectively. Figures $6 \mathrm{~B}$ and $6 \mathrm{D}$ show representative quantitative image analyses of bacteria (spheres) and DNA surface (green). Bacteria that are within $1 \mu \mathrm{m}$ of

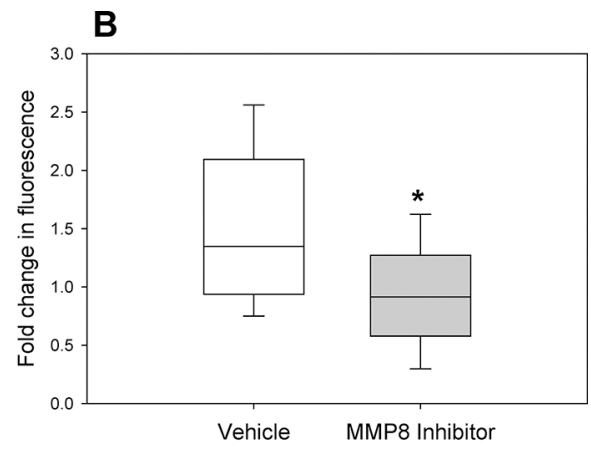

Figure 4. Phagocytic activity of peritoneal macrophages from juvenile MMP8 null mice versus wild-type mice. (A) Pooled primary peritoneal macrophages of MMP8 null juvenile mice took up fewer fluorescent-labeled $E$. coli compared with wild-type pooled peritoneal macrophages. (B) Pooled primary macrophages from wild-type mice treated ex vivo with an MMP8 inhibitor took up fewer fluorescent-labeled E. coli compared with wild-type macrophages treated with the DMSO vehicle. Data are plotted as fold change relative to wild-type macrophages exposed to fluorescent bacteria in cell culture media. ${ }^{*} p<0.05$ by 2 -tailed sum rank test; 3 separate experiments. the DNA surface are considered affiliated with the DNA surface (magenta), whereas bacteria farther than $1 \mu \mathrm{m}$ from the DNA surface are not considered affiliated with the DNA surface (aqua). For the wild-type NETs (Figure 6B) the majority of bacteria were affiliated with the DNA surface. In contrast, for the MMP8 null NETs (Figure 6D), a smaller proportion of bacteria were affiliated with the DNA surface. Figure $6 \mathrm{E}$ shows that, on average, NETs formed by MMP8 null mice were half as efficient at capturing bacteria when compared with wild-type NETs. These data demonstrate that absence of MMP8 decreases NET efficiency and suggests an additional potential mechanism accounting for the observed decreased bacterial clearance in MMP8 null juvenile mice.

\section{DISCUSSION}

Genetic ablation of MMP8 decreased survival in juvenile mice with polymicrobial sepsis induced by intraperitoneal injection of cecal slurry. In contrast, our previous studies showed that adult MMP8 null mice had either no change in sepsis survival compared with wildtype (cecal slurry), or a sepsis survival advantage (cecal ligation and puncture) (15). Collectively, these data support the concept that developmental age strongly influences the host response to sepsis $(5,18-20)$. The differing phenotypes between adult and juvenile mice following cecal slurry injections also underscores the need to consider developmental age-specific therapies.

The mechanism leading to increased mortality in the juvenile MMP8 null mice seems to involve reduced bacterial clearance due to reduced phagocytosis and reduced NET efficiency. Previous research identified a role for MMP8 in chemotaxis of neutrophils and macrophages, the primary phagocytic cells of the innate immune system $(12,14,21)$. Since early life host defense is predominantly supported by the innate immune system, we compared qualitative and functional endpoints of neutrophil and macrophage function between juvenile MMP8 null and wild-type mice (16). 

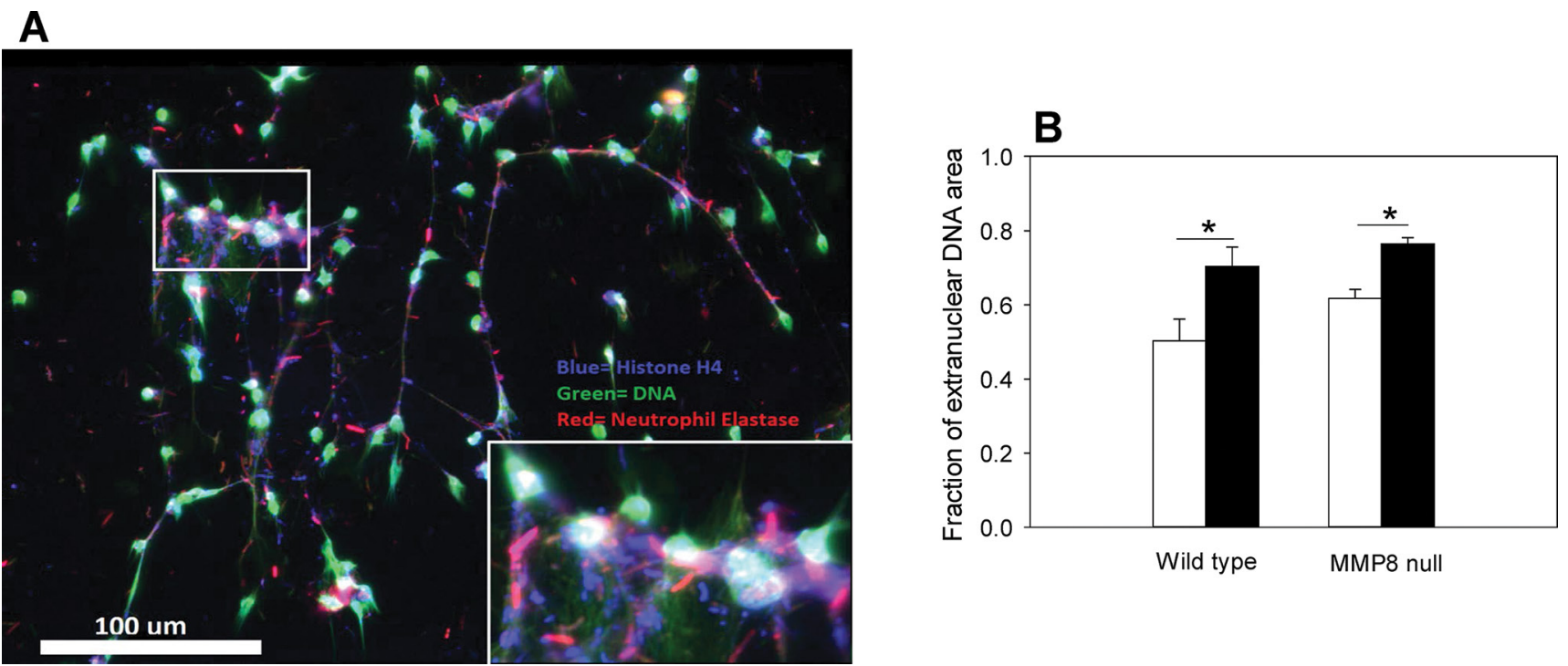

Figure 5. Net formation after cecal slurry. (A) Representative image from wild-type animals treated with cecal slurry. Histone H4 (blue) co-localized with DNA (green), with neutrophil elastase (red) present both in degenerating neutrophils and along DNA NETs. (B) Six hours after cecal slurry, both wild-type and MMP null mice had an increased percentage of DNA signal outside the nucleus; however, there was no difference between wild-type $(n=10)$ and MMP8 null animals $(n=10) .{ }^{*} p<0.05$ versus respective sham injected animals. All data reported as mean \pm SEM. White bars represent sham-injected mice and black bars represent slurry-injected mice.

A

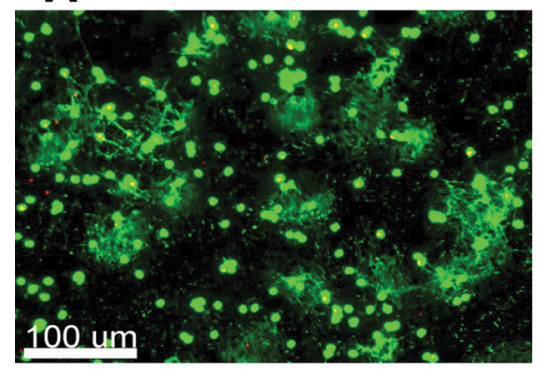

C

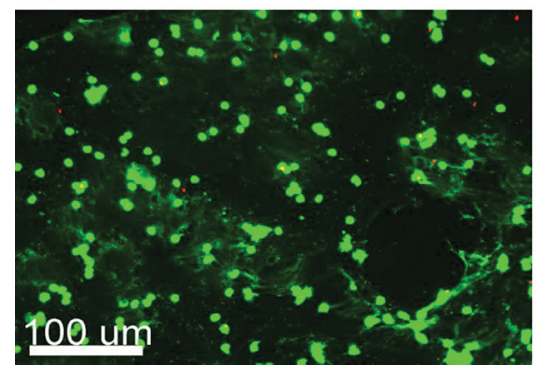

B

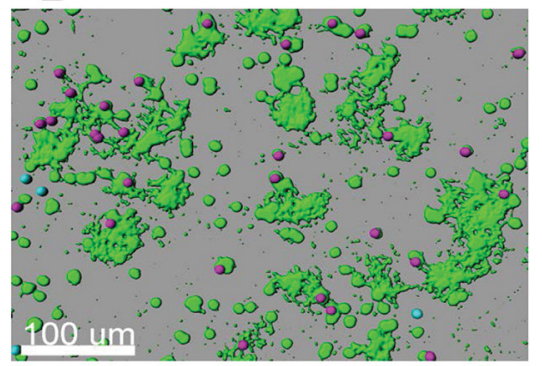

D

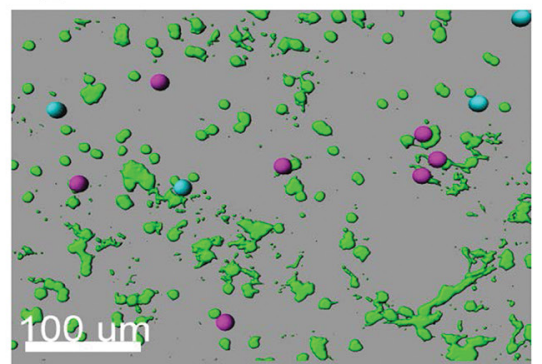

$\mathbf{E}$

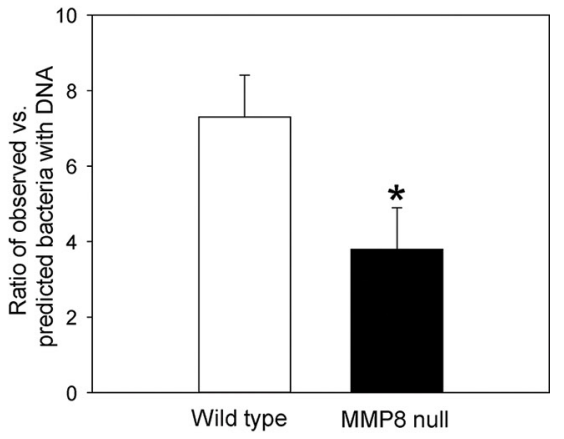

Figure 6. E. coli adherence to NETs of MMP8 null and wild-type animals. (A) Qualitatively, WT animals demonstrated frequent adherence of fluorescent-labeled bacteria to NETs. (B) By quantitative image analysis, the majority of bacteria (spheres) in WT nets were within $1 \mu \mathrm{m}$ of the DNA surface (green). Bacteria co-localized with DNA are labeled magenta and non-co-localized bacteria as aqua. (C) MMP8 null animals demonstrate less frequent localization of bacteria with NETS. (D) Non-co-localization of bacteria was more frequent in MMP8 null samples. (E) The bacteria in MMP8 null animals $(n=10)$ were about $50 \%$ less likely to be associated with DNA compared with the bacteria in WT animals $(n=10) .{ }^{*} p<0.05$ by 2 -tailed sum rank test. All data reported as mean \pm SEM. 
When comparing the peritoneal fluid of MMP8 null and wild-type mice, there was a trend toward a greater number of neutrophils, macrophages and total white blood cells in the peritoneal fluid of MMP8 null mice. Thus, recruitment of phagocytic cells per se does not appear to be impaired in the absence of MMP8.

Absolute cytokine concentrations were greater in the peritoneal fluid of MMP8 null mice, but this difference was eliminated after correction for total protein content in the peritoneal space. While the results are consistent with a more robust cytokine response in MMP8 null animals, an alternative interpretation is that they reflect greater disruption of the peritoneal membrane and greater vascular leak among juvenile MMP8 null mice challenged with cecal slurry injections. In either case, the local inflammatory response of MMP8 juvenile mice seems to be more pronounced compared with wild-type juvenile mice. This might reflect an ongoing bacterial burden. The finding that CXCL1 remained increased after correction for protein concentration in MMP8 null mice supports this assertion.

The relationship between $M M P 8$ and bacterial clearance appears to be related to cellular function rather than cellular recruitment or the cytokine milieu. Both phagocytic function and NET function were impaired in the MMP8 null juvenile mice. In addition, ex vivo inhibition of MMP8 activity decreased the phagocytic capability of wild-type peritoneal macrophages. There was no difference in quantitative NET formation between MMP8 null and wildtype mice, but MMP8 null NETs were less efficient at capturing bacteria. Together, these phagocytic impairments may lead to an increased bacterial burden and increased mortality in MMP8 null juvenile mice after cecal slurry. The ability of antibiotics to rescue MMP8 null mice further supports this assertion. Thus, MMP8 seems to play a functional role in the innate immune response of juvenile mice subjected to cecal slurry.
The unique phenotype of MMP8 null juvenile mice supports previous findings that developmental age influences the host response to sepsis (5). The neonatal mouse relies primarily on the innate immune system, which makes it more vulnerable to any genetic defects in phagocytic activity (22). Older animals with a competent adaptive immune system may be better equipped to compensate for any impairment of the innate system and therefore exhibit a different phenotype in response to genetic ablation or pharmacologic inhibition of MMP8 (15). Identifying age-specific alterations in the host response is a critical step in designing developmental age-appropriate therapies for sepsis. Many attempts at reducing the incidence, morbidity and mortality associated with neonatal sepsis have failed due to an underappreciation of how the immature host response differs from that of adults $(23,24)$. Identification of critical components of the neonatal and juvenile immune systems will allow us to more specifically augment the age-appropriate host component as therapy for sepsis (25).

The major limitation of our study is that we have yet to delineate the exact mechanism by which MMP8 enhances phagocytosis and NET function. One study of adult human NETs identified MMP8 along the length of the NET; however, we were unable to consistently co-localize MMP8 along the juvenile murine NETs (26). This difference may be due to differences in antibody sensitivity or in murine and human NET composition and structure. Alternatively, it may reflect the influence of developmental age on MMP8 and its role in NET formation. Furthermore, a genome-wide study of children with septic shock demonstrated that there are multiple developmental age groups within the juvenile population that need to be studied separately (5).

Another limitation involves our inability to determine if non-neutrophil sources of MMP8 contributed to our observations in this juvenile model of murine sepsis. In our previous study, we identified the intestine as a critical source of MMP8 in an adult model of murine sepsis (15). Due to size limitations, we were unable to conduct analogous experiments in our juvenile model.

\section{CONCLUSION}

In summary, we found that in a juvenile model of polymicrobial sepsis, MMP8 contributes to clearance of bacteria from the intraperitoneal cavity, and consequently, absence of MMP8 negatively influences survival. MMP8 appears to contribute to bacterial clearance through defects in both phagocytosis and NET function. The exact mechanisms by which MMP8 contributes to these processes require further exploration. These findings appear to be unique to the juvenile host septic response, because adult mice have a survival advantage when MMP8 is either genetically ablated or inhibited pharmacologically; adult mice benefit from MMP8 inhibition, whereas this same strategy is harmful in juvenile mice. This illustrates the need to consider developmental influences and differences when contemplating novel sepsis therapies.

\section{DISCLOSURE}

The authors declare that they have no competing interests as defined by Molecular Medicine, or other interests that might be perceived to influence the results and discussion reported in this paper.

\section{REFERENCES}

1. Lagu T, Rothberg MB, Shieh MS, Pekow PS, Steingrub JS, Lindenauer PK. Hospitalizations, costs, and outcomes of severe sepsis in the united states 2003 to 2007. Crit. Care Med. 2012;40:754-61.

2. Watson RS, Carcillo JA. Scope and epidemiology of pediatric sepsis. Pediatr. Crit. Care Med. 2005;6:S3-5.

3. Dellinger RP, et al. Surviving sepsis campaign: International guidelines for management of severe sepsis and septic shock: 2012. Crit. Care Med. 2013;41:580-637.

4. Wong HR, et al. Genome-level expression profiles in pediatric septic shock indicate a role for altered zinc homeostasis in poor outcome. Physiol. Genomics. 2007;30:146-55.

5. Wynn JL, et al. The influence of developmental age on the early transcriptomic response of children with septic shock. Mol. Med. 2011;17:1146-56. 
6. Cvijanovich $\mathrm{N}$, et al. Validating the genomic signature of pediatric septic shock. Physiol. Genomics 2008;34:127-34.

7. Shanley TP, et al. Genome-level longitudinal expression of signaling pathways and gene networks in pediatric septic shock. Mol. Med. 2007;13:495-508

8. Solan PD, Dunsmore KE, Denenberg AG, Odoms K, Zingarelli B, Wong HR. A novel role for matrix metalloproteinase-8 in sepsis. Crit. Care Med. 2012;40:379-87.

9. Wong HR, et al. The pediatric sepsis biomarker risk model. Crit. Care. 2012;16:R174.

10. Rella JM, Jilma B, Fabry A, Kaynar AM, Mayr FB. Mmp- 8 genotypes influence the inflammatory response in human endotoxemia. Inflammation. 2014;37:451-56

11. Wang $\mathrm{H}$, et al. Functionally significant snp mmp8 promoter haplotypes and preterm premature rupture of membranes (pprom). Hum. Mol. Genet. 2004;13:2659-69.

12. Tester AM, et al. Lps responsiveness and neutrophil chemotaxis in vivo require pmn mmp-8 activity. PLoS One. 2007;2:e312.

13. Van Lint P, Libert C. Chemokine and cytokine processing by matrix metalloproteinases and its effect on leukocyte migration and inflammation. J. Leukoc. Biol. 2007;82:1375-81.

14. Quintero PA, Knolle MD, Cala LF, Zhuang Y, Owen CA. Matrix metalloproteinase-8 inactivates macrophage inflammatory protein-1 alpha to reduce acute lung inflammation and injury in mice. J. Immunol. 2010;184:1575-88.

15. Atkinson SJ, et al. Intestine-derived matrix metalloproteinase- 8 is a critical mediator of polymicrobial peritonitis. Crit. Care Med. 2016;44:e200-6

16. Wynn JL, et al. Defective innate immunity predisposes murine neonates to poor sepsis outcome but is reversed by thr agonists. Blood. 2008;112:1750-58.

17. Committee for the Update of the Guide for the Care and Use of Laboratory Animals, Institute for Laboratory Animal Research, Division on Earth and Life Studies. (2011) Guide for the Care and Use of Laboratory Animals, 8th Edition. Washington, DC: National Academies Press [cited 2016 July 19].

18. Wynn JL, et al. Increased mortality and altered immunity in neonatal sepsis produced by generalized peritonitis. Shock. 2007;28:675-83.

19. Barton P, et al. Safety, pharmacokinetics, and pharmacodynamics of drotrecogin alfa (activated) in children with severe sepsis. Pediatrics. 2004;113:7-17.

20. Martin GS, Mannino DM, Eaton S, Moss M. The epidemiology of sepsis in the united states from 1979 through 2000. N. Engl. J. Med. 2003;348:1546-54.

21. Van Den Steen PE, Wuyts A, Husson SJ, Proost P, Van Damme J, Opdenakker G. Gelatinase b/mmp-9 and neutrophil collagenase/mmp-8 process the chemokines human gcp-2/cxcl6, ena-78/cxcl5 and mouse gcp-2/lix and modulate their physiological activities. Eur. J. Biochem. 2003;270:3739-49.

22. Silva MT. When two is better than one: Macrophages and neutrophils work in concert in innate immunity as complementary and cooperative partners of a myeloid phagocyte system. J. Leukoc. Biol. 2010;87:93-106.

23. Wynn JL, Neu J, Moldawer LL, Levy O. Potential of immunomodulatory agents for prevention and treatment of neonatal sepsis. J. Perinatol. 2009;29:79-88.

24. Wynn JL, Seed PC, Cotten CM. Does ivig administration yield improved immune function in very premature neonates? J. Perinatol. 2010;30:635-42.

25. Wheeler DS, Wong HR, Zingarelli B. Pediatric sepsis_-part I: "Children are not small adults!" Open Inflamm. J. 2011;4:4-15.

26. Ong CW, et al. Neutrophil-derived mmp-8 drives ampk-dependent matrix destruction in human pulmonary tuberculosis. PLoS Pathog. 2015;11:e1004917.

Cite this article as: Atkinson SJ, et al. (2016) Matrix metalloproteinase- 8 augments bacterial clearance in a juvenile sepsis model. Mol. Med. 22:455-63. 